\title{
A new iterative algorithm for solving some nonlinear prob- lems in Hilbert spaces
}

\author{
T. M. M. Sow
}

Department of Mathematics, Gaston Berger University, Saint Louis, Senegal.

\begin{abstract}
In this paper, a new iterative algorithm for finding a common element of the set of minimizers of a convex function, the set of solutions of variational inequality problem, the set of solutions of equilibrium problems and the set of fixed points of demicontractive mappings is constructed. Convergence theorems are also proved in Hilbert spaces without any compactness assumption. Furthermore, a numerical example is given to demonstrate the implementability of our algorithm. Our theorems are significant improvements in several important recent results.
\end{abstract}

Keywords: Fixed points problem, convex minimization problem, equilibrium problem, variational inequality problem.

2010 MSC: 47H09, 65K05, 47J05.

(C)2020 All rights reserved.

\section{Introduction}

Let $\mathrm{H}$ be a real Hilbert space and $\mathrm{K}$ be a nonempty subset of $\mathrm{H}$. For a set-valued map $A: H \rightarrow 2^{\mathrm{H}}$, the domain of $A, D(A)$, the image of a subset $S$ of $H, A(S)$ the range of $A, R(A)$ and the graph of $A, G(A)$ are defined as follows:

$$
\begin{aligned}
D(A) & :=\{x \in H: A x \neq \emptyset\}, A(S):=\cup\{A x: x \in S\}, \\
R(A) & :=A(H), G(A):=\{[x, u]: x \in D(A), u \in A x\} .
\end{aligned}
$$

An operator $\mathrm{A}: \mathrm{K} \rightarrow \mathrm{H}$ is called monotone if

$$
\langle A x-A y, x-y\rangle_{H} \geqslant 0, \quad \forall x, y \in K .
$$

An operator $\mathrm{A}: \mathrm{K} \rightarrow \mathrm{H}$ is said $\alpha$-inverse strongly monotone if there exists a constant $\alpha>0$ such that

$$
\langle A x-A y, x-y\rangle_{H} \geqslant \alpha\|A x-A y\|^{2}, \quad \forall x, y \in K .
$$

It is immediate that if $A$ is $\alpha$-inverse strongly monotone, then $A$ is monotone and Lipschitz continuous.

Email address: sowthierno89@gmail.com (T. M. M. Sow)

doi: $10.22436 /$ jnsa.013.03.01

Received: 2019-09-02 Revised: 2019-09-28 Accepted: 2019-10-04 
A map $T: K \rightarrow K$ is said to be Lipschitz if there exists an $L \geqslant 0$ such that

$$
\|T x-T y\| \leqslant L\|x-y\|, \quad \forall x, y \in K,
$$

if $\mathrm{L}<1, \mathrm{~T}$ is called contraction and if $\mathrm{L}=1, \mathrm{~T}$ is called non-expansive. We denote by $\operatorname{Fix}(\mathrm{T})$ the set of fixed points of the mapping $T$, that is $\operatorname{Fix}(T):=\{x \in D(T): x=T x\}$. We assume that $\operatorname{Fix}(T)$ is nonempty. If $T$ is non-expansive mapping, it is well known $\operatorname{Fix}(T)$ is closed and convex. A map $T$ is called quasinonexpansive if $\|T x-p\| \leqslant\|x-p\|$ holds for all $x$ in $K$ and $p \in \operatorname{Fix}(T)$. The mapping $T: K \rightarrow K$ is said to be firmly non-expansive, if

$$
\|T x-T y\|^{2} \leqslant\|x-y\|^{2}-\|(x-y)-(T x-T y)\|^{2}, \forall x, y \in K .
$$

A mapping $T: K \rightarrow K$ is called k-strictly pseudo-contractive if there exists $k \in[0,1)$ such that

$$
\|T x-T y\|^{2} \leqslant\|x-y\|^{2}+k\|x-y-(T x-T y)\|^{2}, \quad \forall x, y \in K .
$$

A mapping $T$ is called $k$-demicontractive if $\operatorname{Fix}(T) \neq \emptyset$ and for $k \in[0,1)$, we have

$$
\|T x-p\| \leqslant\|x-p\|+k\|x-T x\|^{2}, \quad \forall x \in K, p \in \operatorname{Fix}(T) .
$$

Remark 1.1. Easily, we obtain the following conclusions:

1. every firmly non-expansive mapping is non-expansive;

2. every non-expansive mapping is quasi-non-expansive;

3. every quasi-non-expansive mapping is 0-demicontractive;

4. every k-strictly pseudo-contractive mapping is $k$-demicontractive.

Fixed point theory is one of the most powerful and important tools of modern mathematics and may be considered a core subject of nonlinear analysis. In the last few decades, the problem of nonlinear analysis with its relation to fixed point theory has emerged as a rapidly growing area of research because of its applications in game theory, optimization problem, control theory, integral and differential equations and inclusions, dynamic systems theory, signal and image processing, and so on. The crucial key of this success is due to the possibility of representing various problems arising in the above disciplines, in the form of an equivalent fixed point problem. Until now there have been many effective algorithms for solving fixed point problem (see, e.g., Yao et al. [35], Chidume [4], Marino et al. [16], Xu [30, 31], and the references therein).

Recall the classical variational inequality problem is to find a find $u \in K$ such that

$$
\langle A u, v-u\rangle \geqslant 0, \forall v \in K \text {. }
$$

We denote the set of solutions of variational inequality problem (1.1) by $\mathrm{VI}(\mathrm{A}, \mathrm{K})$. Variational inequality (1.1) was formulated in the late 1960s by Lions and Stampacchia [11]. Since then, it has been extensively studied via numerical methods. For a lot of real-life problems, such as, in signal processing, resource allocation, image recovery and so on, the constraints can be expressed as the variational inequality problem. Hence, the problem of finding solutions of variational inequality (1.1) has become a flourishing area of contemporary research for numerous mathematicians working in nonlinear operator theory; see, for example, $[12,20,24]$. It is easy to see that $u \in K$ is a solution of variational inequality (1.1) iff $u$ is a fixed point of the mapping $P_{K}(I-\lambda A)$, where $P_{K}$ is the known metric projection from $H$ onto $K, I$ is the identity mapping and $\lambda$ is some real positive number.

A well known method for solving the variational inequality problem is the projection algorithm which starts with $x_{1} \in K$ and generates a sequence $\left\{x_{n}\right\}$ in the following recursion formula,

$$
x_{n+1}=P_{K}\left(x_{n}-\lambda_{n} A x_{n}\right), n \geqslant 1,
$$


where $\left\{\lambda_{n}\right\}$ is a sequence of positive numbers satisfying appropriate conditions. In the case that $A$ is $\alpha$-inverse strongly monotone, Iiduka et al. [9] proved that the sequence $\left\{x_{n}\right\}$ generated by (1.2) converges weakly to an element of $\mathrm{VI}(A, K)$. Spotlights have been shed on the modification of the above projection algorithm so that the norm convergence is guaranteed under mild conditions recent; see $[2,27,28]$.

Equilibrium problems were originally studied in [3] as a unifying class of variational problems. Given a nonempty set $K$, and a bifunction $g: K \times K \rightarrow \mathbb{R}$. The equilibrium problem $\operatorname{EP}(K, g)$ is formulated as follows:

$$
\text { Find a point } \mathrm{x}^{*} \in \mathrm{K} \text { such that } \mathrm{g}\left(\mathrm{x}^{*}, \mathrm{y}\right) \geqslant 0 \text { for every } \mathrm{y} \in \mathrm{K} \text {. }
$$

The set of all solutions of $\operatorname{EP}(K, g)$ is denoted by $\operatorname{EP}(g)$. Typical studies for $\operatorname{EP}(K, g)$ are extensively carried out in Hilbert spaces and recently in real Banach spaces. The set of solutions is denoted by $\operatorname{EP}(\mathrm{g})$. Equilibrium problems have had a great impact and influence on the development of several branches of pure and applied sciences. It has been shown that the equilibrium problem theory provides a novel and unified treatment of a wide class of problems which arise in economics, finance, image reconstruction, ecology, transportation, network, elasticity, and optimization. It has been shown $[22,33]$ that equilibrium, problems include variational inequalities, minimax inequalities, the Nash equilibrium, and game theory as special cases. Recently, a lot of iterative algorithms have been studied in infinite dimensional spaces, see $[3,21]$ and the references therein.

The minimization problem (MP) is one of the most important problems in nonlinear analysis and optimization theory. The MP is defined as follows: find $x \in H$, such that

$$
F(x)=\min _{y \in H} F(y),
$$

where $\mathrm{F}: \mathrm{H} \rightarrow(-\infty,+\infty]$ is a proper convex and lower semi-continuous. The set of all minimizers of $F$ on $H$ is denoted by $\operatorname{argmin}_{y \in H} F(y)$. A successful and powerful tool for solving this problem is the well-known proximal point algorithm (shortly, the PPA) which was initiated by Martinet [18] in 1970 and later studied by Rockafellar [23] in 1976. Let $\mathrm{H}$ be a real Hilbert space and $\mathrm{F}: \mathrm{H} \rightarrow(-\infty,+\infty$ ] be a proper lower semi-continuous and convex function. The PPA is defined as follows:

$$
\left\{\begin{array}{l}
x_{1} \in H, \\
x_{n+1}=\operatorname{argmin}_{y \in H}\left[F(y)+\frac{1}{2 \lambda_{n}}\left\|x_{n}-y\right\|^{2}\right],
\end{array}\right.
$$

where $\lambda_{n}>0$ for all $n \geqslant 1$. In [23] Rockafellar proved that the sequence $\left\{x_{n}\right\}$ given by (1.3) converges weakly to a minimizer of $F$. He then posed the following question

(Q1): does the sequence $\left\{x_{n}\right\}$ converges strongly?

This question was resolved in the negative by Güler [8] (1991). He produced a proper lower semi continuous and convex function $\mathrm{F}$ in $l_{2}$ for which the PPA converges weakly but not strongly. This leads naturally to the following question:

(Q2): can the PPA be modified to guarantee strong convergence?

In response to Q2, several works have been done (see, e.g., Güler [8], Kamimura and Takahashi [10], Chidume and Djitte [5] and the references therein). In the recent years, the problem of finding a common element of the set of solutions of various convex minimization problems and the set of fixed points for nonlinear mapping in the framework of Hilbert spaces and Banach spaces have been intensively studied by many authors.

Motivated and inspired by the ongoing results in this field, we introduce a new iterative algorithm for approximating a common element to the set of minimizers of proper lower semicontinuous convex function, the set of solutions of variational inequality problem, the set of solutions of equilibrium problems and the set of fixed points of demicontractive mappings in Hilbert spaces. Then, under appropriate conditions, we establish some strong convergence theorems. The results obtained here extend and unify the result of Takahashi-Takahashi [25], Moudafi [20] and most of the recent results in this direction. Our technique of proof is of independent interest. 


\section{Preliminaries}

Let $\mathrm{K}$ be a nonempty, closed convex subset of $\mathrm{H}$. The nearest point projection from $\mathrm{H}$ to $\mathrm{K}$, denoted by $P_{K}$ assigns to each $x \in H$ the unique $P_{K} x$ with the property

$$
\left\|x-P_{K} x\right\| \leqslant\|y-x\|
$$

for all $y \in K$. It is well known that $P_{K}$ satisfies

$$
\left\langle x-y, P_{K} x-P_{K} y\right\rangle \geqslant\left\|P_{K} x-P_{K} y\right\|^{2}
$$

and for all $y \in H$ and

$$
\left\langle\mathrm{P}_{\mathrm{K}} z-\mathrm{y}, z-\mathrm{P}_{\mathrm{K}} z\right\rangle \geqslant 0
$$

for all $z \in \mathrm{K}$ and $\mathrm{y} \in \mathrm{H}$.

Remark 2.1. In the context of variational inequality problem (1.1), we have

$$
u \in \mathrm{VI}(\mathrm{A}, \mathrm{K}) \Longleftrightarrow \mathrm{u}=\mathrm{P}_{\mathrm{K}}(\mathrm{I}-\theta \mathrm{A}) \mathrm{u}, \quad \theta>0 .
$$

Lemma 2.2 ([23, Demiclosedness principle]). Let $\mathrm{H}$ be a real Hilbert space, $\mathrm{K}$ be a closed convex subset of $\mathrm{H}$, and $\mathrm{T}: \mathrm{K} \rightarrow \mathrm{K}$ be a nonexpansive mapping. Then $\mathrm{I}-\mathrm{T}$ is demiclosed; that is,

$$
\left\{x_{n}\right\} \subset K, x_{n} \rightarrow x \in K \text { and }(I-T) x_{n} \rightarrow y \text { implies that }(\mathrm{I}-\mathrm{T}) \mathrm{x}=\mathrm{y} .
$$

Lemma 2.3 ([4]). Let $\mathrm{H}$ be a real Hilbert space. Then for any $\mathrm{x}, \mathrm{y} \in \mathrm{H}$, the following inequalities hold:

$$
\begin{aligned}
\|x+y\|^{2} & \leqslant\|x\|^{2}+2\langle y, x+y\rangle, \\
\|\lambda x+(1-\lambda) y\|^{2} & =\lambda\|x\|^{2}+(1-\lambda)\|y\|^{2}-(1-\lambda) \lambda\|x-y\|^{2}, \lambda \in(0,1) .
\end{aligned}
$$

Lemma 2.4 ([31]). Assume that $\left\{a_{n}\right\}$ is a sequence of nonnegative real numbers such that $a_{n+1} \leqslant\left(1-\alpha_{n}\right) a_{n}+$ $\alpha_{n} \sigma_{n}$ for all $n \geqslant 0$, where $\left\{\alpha_{n}\right\}$ is a sequence in $(0,1)$ and $\left\{\sigma_{n}\right\}$ is a sequence in $\mathbb{R}$ such that

(a) $\sum_{n=0}^{\infty} \alpha_{n}=\infty$;

(b) $\limsup _{n \rightarrow \infty} \sigma_{n} \leqslant 0$ or $\sum_{n=0}^{\infty}\left|\sigma_{n} \alpha_{n}\right|<\infty$.

Then $\lim _{n \rightarrow \infty} a_{n}=0$.

Lemma 2.5 ([17]). Assume $\mathrm{K}$ is a closed convex subset of a Hilbert space $\mathrm{H}$. Let $\mathrm{T}: \mathrm{K} \rightarrow \mathrm{K}$ be a self-mapping of $\mathrm{K}$. If $\mathrm{T}$ is a $\mathrm{k}$-demicontractive mapping, then the fixed point set $\operatorname{Fix}(\mathrm{T})$ is closed and convex.

Lemma 2.6 ([17]). Let $\mathrm{K}$ be a nonempty closed convex subset of a real Hilbert space $\mathrm{H}$ and $\mathrm{T}: \mathrm{K} \rightarrow \mathrm{K}$ be a mapping.

(i) If $\mathrm{T}$ is a $\mathrm{k}$-strictly pseudo-contractive mapping, then $\mathrm{T}$ satisfies the Lipschitzian condition

$$
\|T x-T y\| \leqslant \frac{1+k}{1-k}\|x-y\| .
$$

(ii) If $\mathrm{T}$ is a $\mathrm{k}$-strictly pseudo-contractive mapping, then the mapping $\mathrm{I}-\mathrm{T}$ is demiclosed at 0.

Lemma 2.7 ([23]). Let $\mathrm{K}$ be a nonempty closed and convex subset of a real Hilbert space $\mathrm{H}$ and $\mathrm{A}$ is a monotone, hemicontinuous map of $\mathrm{K}$ into $\mathrm{H}$. Let $\mathrm{B} \subset \mathrm{H} \times \mathrm{H}$ be an operator defined as follows:

$$
\mathrm{B} z= \begin{cases}\mathrm{A} z+\mathrm{N}_{\mathrm{K}}(z), & \text { if } z \in \mathrm{K}, \\ \emptyset, & \text { if } z \notin \mathrm{K},\end{cases}
$$

where $\mathrm{N}_{\mathrm{K}}(z)$ is the normal $\mathrm{K}$ at $z$ and is defined as follows:

$$
\mathrm{N}_{\mathrm{K}}(z)=\{w \in \mathrm{H}:\langle w, z-v\rangle \geqslant 0, \forall v \in \mathrm{K}\} .
$$

Then, $\mathrm{B}$ is maximal monotone and $\mathrm{B}^{-1}(0)=\mathrm{VI}(\mathrm{A}, \mathrm{K})$. 
Lemma 2.8 ([24]). Let $\mathrm{H}$ be a real Hilbert space and $\mathrm{K}$ be a nonempty, closed convex subset of $\mathrm{H}$. Let $\mathrm{A}: \mathrm{K} \rightarrow \mathrm{H}$ be an $\alpha$-inverse strongly monotone mapping. Then, $\mathrm{I}-\theta \mathrm{A}$ is nonexpansive mapping for all $\mathrm{x}, \mathrm{y} \in \mathrm{K}$ and $\theta \in[0,2 \alpha]$.

Lemma 2.9 ([13]). Let $t_{n}$ be a sequence of real numbers that does not decrease at infinity in a sense that there exists a subsequence $t_{n_{i}}$ of $t_{n}$ such that $t_{n_{i}}$ such that $t_{n_{i}} \leqslant t_{n_{i+1}}$ for all $i \geqslant 0$. For sufficiently large numbers $n \in \mathbb{N}$, an integer sequence $\{\tau(\mathrm{n})\}$ is defined as follows:

$$
\tau(n)=\max \left\{k \leqslant n: t_{k} \leqslant t_{k+1}\right\} .
$$

Then, $\tau(\mathrm{n}) \rightarrow \infty$ as $\mathrm{n} \rightarrow \infty$ and

$$
\max \left\{t_{\tau(n)}, t_{n}\right\} \leqslant t_{\tau(n)+1} .
$$

Let $\mathrm{F}: \mathrm{K} \rightarrow(-\infty,+\infty]$ be a proper convex and lower semi-continuous function. For any $\lambda>0$, define the Moreau-Yosida resolvent of $F$ in a real Hilbert space $H$ as follows:

$$
\mathrm{J}_{\lambda}^{\mathrm{F}} \mathrm{x}=\operatorname{argmin}_{\mathfrak{u} \in \mathrm{K}}\left[\mathrm{F}(\mathrm{u})+\frac{1}{2 \lambda}\|x-u\|^{2}\right],
$$

for all $x \in H$. It was shown in [8] that the set of fixed points of the resolvent associated with $F$ coincides with the set of minimizers of $F$. Also, the resolvent $J_{\lambda}^{F}$ of $F$ is non-expansive for all $\lambda>0$.

Lemma 2.10 ([19])). For any $r>0$ and $\mu>0$, the following holds:

$$
\mathrm{J}_{\mathrm{r}}^{\mathrm{F}} x=\mathrm{J}_{\mu}^{\mathrm{F}} x\left(\frac{\mu}{\mathrm{r}} x+\left(1-\frac{\mu}{\mathrm{r}}\right) \mathrm{J}_{\mathrm{r}}^{\mathrm{F}} x\right) \text {. }
$$

Lemma 2.11 ([13, Sub-differential inequality]). Let $\mathrm{F}: \mathrm{H} \rightarrow(-\infty,+\infty]$ be a proper convex and lower semicontinuous function. Then, for all $\mathrm{x}, \mathrm{y} \in \mathrm{H}$ and $\lambda>0$, the following sub-differential inequality holds:

$$
\frac{1}{\lambda}\left\|J_{\lambda}^{F} x-y\right\|^{2}-\frac{1}{\lambda}\|x-y\|^{2}+\frac{1}{\lambda}\left\|x-J_{\lambda}^{F} x\right\|^{2}+F\left(J_{\lambda}^{F} x\right) \leqslant F(y) .
$$

For solving the equilibrium problem for a bifunction $g: K \times K \rightarrow \mathbb{R}$, let us assume that $g$ satisfies the following conditions:

(A1) $g(x, x)=0$ for all $x \in K$;

(A2) $g$ is monotone, i.e., $g(x, y)+g(y, x) \leqslant 0$ for all $x, y \in K$;

(A3) for each $x, y, z \in K$,

$$
\lim _{t \rightarrow 0} g(t z+(1-t) x, y) \leqslant g(x, y)
$$

(A4) for each $x \in K, \quad y \rightarrow g(x, y)$ is convex and lower semicontinuous.

The following lemma appears implicitly in [3].

Lemma 2.12 ([3]). Let $\mathrm{K}$ be a nonempty closed convex subset of $\mathrm{H}$ and let $\mathrm{g}$ be a bifunction of $\mathrm{K} \times \mathrm{K}$ into $\mathbb{R}$ satisfying (A1)-(A4). Let $\mathrm{r}>0$ and $\mathrm{x} \in \mathrm{H}$. Then, there exists $z \in \mathrm{K}$ such that

$$
g(z, y)+\frac{1}{r}\langle y-z, z-x\rangle \geqslant 0, \forall y \in K .
$$

The following lemma was given in [32].

Lemma 2.13 ([32]). Assume that $\mathrm{g}: \mathrm{K} \times \mathrm{K} \rightarrow \mathbb{R}$ satisfying (A1)-(A4). For $\mathrm{r}>0$ and $\mathrm{x} \in \mathrm{H}$, define a mapping $\mathrm{T}_{\mathrm{r}}: \mathrm{H} \rightarrow \mathrm{K}$ as follows

$$
\mathrm{T}_{\mathrm{r}}(x)=\left\{z \in \mathrm{K}, \mathrm{g}(z, \mathrm{y})+\frac{1}{\mathrm{r}}\langle\mathrm{y}-z, z-x\rangle \geqslant 0, \quad \forall \mathrm{y} \in \mathrm{K}\right\},
$$

for all $\mathrm{x} \in \mathrm{H}$. Then, the following hold:

1. $\mathrm{T}_{\mathrm{r}}$ is single-valued;

2. $\mathrm{T}_{\mathrm{r}}$ is firmly non-expansive, i.e., $\left\|\mathrm{T}_{\mathrm{r}}(\mathrm{x})-\mathrm{T}_{\mathrm{r}}(\mathrm{y})\right\|^{2} \leqslant\left\langle\mathrm{~T}_{\mathrm{r}} \mathrm{x}-\mathrm{T}_{\mathrm{r}} \mathrm{y}, \mathrm{x}-\mathrm{y}\right\rangle$ for any $\mathrm{x}, \mathrm{y} \in \mathrm{H}$;

3. $\operatorname{Fix}\left(\mathrm{T}_{\mathrm{r}}\right)=\mathrm{EP}(\mathrm{g})$;

4. $\mathrm{EP}(\mathrm{g})$ is closed and convex. 


\section{Main result}

Now, we prove our main result.

Theorem 3.1. Let $\mathrm{K}$ be a nonempty, closed convex subset of a real Hilbert space $\mathrm{H}$. Let $\mathrm{A}: \mathrm{K} \rightarrow \mathrm{H}$ be an $\alpha$-inverse strongly monotone operator and let $\mathrm{g}$ be a bifunction from $\mathrm{K} \times \mathrm{K} \rightarrow \mathbb{R}$ satisfying (A1)-(A4). Let $\mathrm{f}: \mathrm{K} \rightarrow \mathrm{K}$ be a contraction with coefficient $\mathrm{b}$ and $\mathrm{F}: \mathrm{K} \rightarrow(-\infty,+\infty]$ be a proper convex and lower semi-continuous function. Let $\mathrm{T}: \mathrm{K} \rightarrow \mathrm{K}$ be a $\lambda$-demicontractive mapping such that $\Gamma:=\operatorname{Fix}(\mathrm{T}) \cap \mathrm{VI}(\mathrm{A}, \mathrm{K}) \cap \operatorname{argmin}_{\mathrm{u} \in \mathrm{K}} \mathrm{F}(\mathrm{u}) \cap \mathrm{EP}(\mathrm{g}) \neq \emptyset$ and $\mathrm{I}-\mathrm{T}$ is demiclosed at the origin. Let $\left\{x_{\mathrm{n}}\right\}$ be a sequence defined as follows:

$$
\left\{\begin{array}{l}
x_{0} \in K, \\
u_{n}=\operatorname{argmin}_{u \in K}\left[F(u)+\frac{1}{2 \lambda_{n}}\left\|u-x_{n}\right\|^{2}\right], \\
z_{n}=P_{K}\left(I-\theta_{n} A\right) u_{n}, \\
g\left(v_{n}, y\right)+\frac{1}{r_{n}}\left\langle y-v_{n}, v_{n}-z_{n}\right\rangle \geqslant 0, \forall y \in K, \\
y_{n}=\beta_{n} v_{n}+\left(1-\beta_{n}\right) T v_{n}, \\
x_{n+1}=\alpha_{n} f\left(x_{n}\right)+\left(1-\alpha_{n}\right) y_{n},
\end{array}\right.
$$

where $\left.\beta_{n} \in\right] \lambda, 1\left[,\left\{\alpha_{n}\right\} \subset(0,1),\left\{\theta_{n}\right\} \subset(0,1)\right.$ and $\left.\left\{r_{n}\right\} \subset\right] 0, \infty$ [ satisfy the following conditions; $\lim _{n \rightarrow \infty} \alpha_{n}=0$, $\sum_{n=0}^{\infty} \alpha_{n}=\infty, \lim _{n \rightarrow \infty} \inf \left(1-\beta_{n}\right)\left(\beta_{n}-\lambda\right)>0, \theta_{n} \in[a, b] \subset(0, \min \{1,2 \alpha\}), \lim _{n \rightarrow \infty} \inf r_{n}>0$ and $\left\{\lambda_{n}\right\}$ is a sequence such that $\lambda_{n} \geqslant \beta>0$ for all $n \geqslant 1$ and some $\beta$. Then, the sequence $\left\{x_{n}\right\}$ generated by (3.1) converges strongly to a point $x^{*} \in \Gamma$, where $x^{*}$ is the unique solution of the variational inequality:

$$
\left\langle x^{*}-f\left(x^{*}\right), x^{*}-p\right\rangle \leqslant 0, \quad \forall p \in \Gamma .
$$

Proof. From $(\mathrm{I}-\mathrm{f})$ being strongly monotone and $\Gamma$ is closed convex, then the variational inequality (3.2) has a unique solution in $\Gamma$. In what follows, we denote $x^{*}$ to be the unique solution of (3.2). Now we show that $\left\{x_{n}\right\}$ is bounded. Let $p \in \Gamma$, then $F(p) \leqslant F(u)$ for all $u \in K$. This implies that

$$
F(p)+\frac{1}{2 \lambda_{n}}\|p-p\|^{2} \leqslant F(u)+\frac{1}{2 \lambda_{n}}\|u-p\|^{2}
$$

and hence $J_{\lambda_{n}}^{F} p=p$ for all $n \geqslant 1$, where $J_{\lambda_{n}}^{F}$ is the Moreau-Yosida resolvent of $F$ in K. From (2.2), $v_{\mathrm{n}}=\mathrm{T}_{\mathrm{r}_{\mathrm{n}}} z_{\mathrm{n}}$, and Lemma 2.8, we have

$$
\left\|v_{n}-p\right\|=\left\|T_{r_{n}} z_{n}-p\right\| \leqslant\left\|z_{n}-p\right\|=\left\|P_{K}\left(I-\theta_{n} A\right) u_{n}-p\right\| \leqslant\left\|J_{\lambda_{n}}^{F} x_{n}-p\right\| \leqslant\left\|x_{n}-p\right\|, \quad \forall n \geqslant 0 .
$$

Applying Lemma 2.3, we have

$$
\begin{aligned}
\left\|y_{n}-p\right\|^{2} & =\left\|\beta_{n}\left(v_{n}-p\right)+\left(1-\beta_{n}\right)\left(T v_{n}-p\right)\right\|^{2} \\
& =\beta_{n}\left\|v_{n}-p\right\|^{2}+\left(1-\beta_{n}\right)\left\|T v_{n}-p\right\|^{2}-\beta_{n}\left(1-\beta_{n}\right)\left\|T v_{n}-v_{n}\right\|^{2} .
\end{aligned}
$$

Using the fact that $\mathrm{T}$ is $\lambda$-demicontractive, we obtain

$$
\left\|y_{n}-p\right\|^{2} \leqslant \beta_{n}\left\|v_{n}-p\right\|^{2}+\left(1-\beta_{n}\right)\left(\left\|v_{n}-p\right\|^{2}+\lambda\left\|T v_{n}-v_{n}\right\|^{2}\right)-\beta_{n}\left(1-\beta_{n}\right)\left\|T v_{n}-v_{n}\right\|^{2} .
$$

Hence

$$
\left\|y_{n}-p\right\|^{2} \leqslant\left\|v_{n}-p\right\|^{2}-\left(1-\beta_{n}\right)\left(\beta_{n}-\lambda\right)\left\|T v_{n}-v_{n}\right\|^{2}
$$

Since $\left.\beta_{n} \in\right] \lambda, 1[$, we have

$$
\left\|y_{n}-p\right\| \leqslant\left\|v_{n}-p\right\| \leqslant\left\|z_{n}-p\right\| \leqslant\left\|u_{n}-p\right\| \leqslant\left\|x_{n}-p\right\|, \quad \forall n \geqslant 0 .
$$


By using (3.1) and (3.3), we obtain

$$
\begin{aligned}
\left\|x_{n+1}-p\right\| & =\left\|\alpha_{n} f\left(x_{n}\right)+\left(1-\alpha_{n}\right) y_{n}-p\right\| \\
& \leqslant \alpha_{n}\left\|f\left(x_{n}\right)-f(p)\right\|+\left(1-\alpha_{n}\right)\left\|y_{n}-p\right\|+\alpha_{n}\|f(p)-p\| \\
& \leqslant\left(1-\alpha_{n}(1-b)\right)\left\|x_{n}-p\right\|+\alpha_{n}\|f(p)-p\| \\
& \leqslant \max \left\{\left\|x_{n}-p\right\|, \frac{\|f(p)-p\|_{1}}{1-b}\right\} .
\end{aligned}
$$

By induction, we conclude that

$$
\left\|x_{n}-p\right\| \leqslant \max \left\{\left\|x_{0}-p\right\|, \frac{\|f(p)-p\|}{1-b}\right\}, \quad n \geqslant 1 .
$$

Hence $\left\{x_{n}\right\}$ is bounded, also $\left\{y_{n}\right\},\left\{A x_{n}\right\},\left\{u_{n}\right\}$, and $\left\{f\left(x_{n}\right)\right\}$ are all bounded. Thus we have

$$
\begin{aligned}
\left\|x_{n+1}-p\right\|^{2} \leqslant & \left\|\alpha_{n} f\left(x_{n}\right)+\left(1-\alpha_{n}\right) y_{n}-p\right\|^{2} \\
\leqslant & \alpha_{n}^{2}\left\|f\left(x_{n}\right)-p\right\|^{2}+\left(1-\alpha_{n}\right)^{2}\left\|y_{n}-p\right\|^{2}+2 \alpha_{n}\left(1-\alpha_{n}\right)\left\|f\left(x_{n}\right)-p\right\|\left\|y_{n}-p\right\| \\
\leqslant & \alpha_{n}^{2}\left\|f\left(x_{n}\right)-p\right\|^{2}+\left(1-\alpha_{n}\right)^{2}\left\|v_{n}-p\right\|^{2}-\left(1-\alpha_{n}\right)^{2}\left(1-\beta_{n}\right)\left(\beta_{n}-\lambda\right)\left\|T v_{n}-v_{n}\right\|^{2} \\
& +2 \alpha_{n}\left(1-\alpha_{n}\right)\left\|f\left(x_{n}\right)-p\right\|\left\|y_{n}-p\right\| .
\end{aligned}
$$

Thus

$$
\begin{gathered}
\left(1-\alpha_{n}\right)^{2}\left(1-\beta_{n}\right)\left(\beta_{n}-\lambda\right)\left\|T v_{n}-v_{n}\right\|^{2} \leqslant \\
\left\|x_{n}-p\right\|^{2}-\left\|x_{n+1}-p\right\|^{2}+\alpha_{n}^{2}\left\|f\left(x_{n}\right)-p\right\|^{2} \\
+2 \alpha_{n}\left(1-\alpha_{n}\right)\left\|f\left(x_{n}\right)-p\right\|\left\|x_{n}-p\right\| .
\end{gathered}
$$

Since $\left\{x_{n}\right\}$ is bounded, then there exists a constant $C>0$ such that

$$
\left(1-\alpha_{n}\right)^{2}\left(1-\beta_{n}\right)\left(\beta_{n}-\lambda\right)\left\|T v_{n}-v_{n}\right\|^{2} \leqslant\left\|x_{n}-p\right\|^{2}-\left\|x_{n+1}-p\right\|^{2}+\alpha_{n} C .
$$

Now we prove that $\left\{x_{n}\right\}$ converges strongly to $x^{*}$. We divide the rest of the proof into two cases.

Case 1. Assume that the sequence $\left\{\left\|x_{n}-p\right\|\right\}$ is monotonically decreasing. Then $\left\{\left\|x_{n}-p\right\|\right\}$ is convergent. Clearly, we have

$$
\lim _{n \rightarrow \infty}\left[\left\|x_{n}-p\right\|^{2}-\left\|x_{n+1}-p\right\|^{2}\right]=0 .
$$

Using the fact that $\lim _{n \rightarrow \infty} \inf \left(1-\beta_{n}\right)\left(\beta_{n}-\lambda\right)>0$, we have

$$
\lim _{n \rightarrow \infty}\left\|v_{n}-T v_{n}\right\|=0
$$

From (3.1), (2.2), convexity of $\|.\|^{2}$, and Lemma 2.8, it follows that

$$
\begin{aligned}
\left\|x_{n+1}-p\right\|^{2} & =\left\|\alpha_{n} f\left(x_{n}\right)+\left(1-\alpha_{n}\right) y_{n}-p\right\|^{2} \\
& \leqslant \alpha_{n}\left\|f\left(x_{n}\right)-p\right\|^{2}+\left(1-\alpha_{n}\right)\left\|y_{n}-p\right\|^{2} \\
& \leqslant \alpha_{n}\left\|f\left(x_{n}\right)-p\right\|^{2}+\left(1-\alpha_{n}\right)\left\|z_{n}-p\right\|^{2} \\
& =\alpha_{n}\left\|f\left(x_{n}\right)-p\right\|^{2}+\left(1-\alpha_{n}\right)\left\|P_{K}\left(I-\theta_{n} A\right) u_{n}-P_{K}\left(I-\theta_{n} A\right) p\right\|^{2} \\
& \leqslant \alpha_{n}\left\|f\left(x_{n}\right)-p\right\|^{2}+\left(1-\alpha_{n}\right)\left[\left\|u_{n}-p\right\|^{2}+\theta_{n}\left(\theta_{n}-2 \alpha\right)\left\|A u_{n}-A p\right\|^{2}\right] \\
& \leqslant \alpha_{n}\left\|f\left(x_{n}\right)-p\right\|^{2}+\left(1-\alpha_{n}\right)\left\|x_{n}-p\right\|^{2}+\left(1-\alpha_{n}\right) a(b-2 \alpha)\left\|A u_{n}-A p\right\|^{2} .
\end{aligned}
$$

Therefore, we have

$$
\left(1-\alpha_{n}\right) a(2 \alpha-b)\left\|A u_{n}-A p\right\|^{2} \leqslant\left\|x_{n}-p\right\|^{2}-\left\|x_{n+1}-p\right\|^{2}+\alpha_{n}\left\|f\left(x_{n}\right)-p\right\|^{2} .
$$


Since, $\alpha_{n} \rightarrow 0$ as $n \rightarrow \infty$ and $\left\{x_{n}\right\}$ is bounded, we obtain

$$
\lim _{n \rightarrow \infty}\left\|A u_{n}-A p\right\|^{2}=0 .
$$

From (2.1) and (3.1), we have

$$
\begin{aligned}
\left\|z_{n}-p\right\|^{2} & =\left\|P_{K}\left(I-\theta_{n} A\right) u_{n}-P_{K}\left(I-\theta_{n} A\right) p\right\|^{2} \\
& \leqslant\left\langle z_{n}-p,\left(I-\theta_{n} A\right) u_{n}-\left(I-\theta_{n} A\right) p\right\rangle \\
& =\frac{1}{2}\left[\left\|\left(I-\theta_{n} A\right) u_{n}-\left(I-\theta_{n} A\right) p\right\|^{2}+\left\|z_{n}-p\right\|^{2}-\left\|\left(I-\theta_{n} A\right) u_{n}-\left(I-\theta_{n} A\right) p-\left(z_{n}-p\right)\right\|^{2}\right] \\
& \leqslant \frac{1}{2}\left[\left\|u_{n}-p\right\|^{2}+\left\|z_{n}-p\right\|^{2}-\left\|u_{n}-z_{n}\right\|^{2}+2 \theta_{n}\left\langle z_{n}-p, A u_{n}-A p\right\rangle-\theta_{n}{ }^{2}\left\|A u_{n}-A p\right\|^{2}\right] \\
& \leqslant \frac{1}{2}\left[\left\|x_{n}-p\right\|^{2}+\left\|z_{n}-p\right\|^{2}-\left\|u_{n}-z_{n}\right\|^{2}+2 \theta_{n}\left\langle z_{n}-p, A u_{n}-A p\right\rangle-\theta_{n}{ }^{2}\left\|A u_{n}-A p\right\|^{2}\right] .
\end{aligned}
$$

So, we obtain

$$
\left\|z_{n}-p\right\|^{2} \leqslant\left\|x_{n}-p\right\|^{2}-\left\|u_{n}-z_{n}\right\|^{2}+2 \theta_{n}\left\langle z_{n}-p, A u_{n}-A p\right\rangle-\theta_{n}{ }^{2}\left\|A u_{n}-A p\right\|^{2},
$$

and thus

$$
\begin{aligned}
\left\|x_{n+1}-p\right\|^{2} \leqslant & \alpha_{n}\left\|f\left(x_{n}\right)-p\right\|^{2}+\left(1-\alpha_{n}\right)\left\|y_{n}-p\right\|^{2} \\
\leqslant & \alpha_{n}\left\|f\left(x_{n}\right)-p\right\|^{2}+\left(1-\alpha_{n}\right)\left\|z_{n}-p\right\|^{2} \\
\leqslant & \alpha_{n}\left\|f\left(x_{n}\right)-p\right\|^{2}+\left\|x_{n}-p\right\|^{2}-\left(1-\alpha_{n}\right)\left\|u_{n}-z_{n}\right\|^{2}-\left(1-\alpha_{n}\right) \theta_{n}{ }^{2}\left\|A u_{n}-A p\right\|^{2} \\
& +2 \theta_{n}\left(1-\alpha_{n}\right)\left\langle z_{n}-p, A u_{n}-A p\right\rangle .
\end{aligned}
$$

By using (3.5) and (3.7), we obtain

$$
\lim _{n \rightarrow \infty}\left\|u_{n}-z_{n}\right\|=0 .
$$

Using Lemma 2.11 and since $F(p) \leqslant F\left(u_{n}\right)$, we get

$$
\left\|x_{n}-u_{n}\right\|^{2} \leqslant\left\|x_{n}-p\right\|^{2}-\left\|u_{n}-p\right\|^{2} .
$$

Therefore, from (3.1), (3.9), and Lemma 2.3, we have

$$
\begin{aligned}
\left\|x_{n+1}-p\right\|^{2} & =\left\|\alpha_{n} f\left(x_{n}\right)+\left(1-\alpha_{n}\right) y_{n}-p\right\|^{2} \\
& =\left\|\alpha_{n}\left(f\left(x_{n}\right)-p\right)+\left(1-\alpha_{n}\right)\left(y_{n}-p\right)\right\|^{2} \\
& \leqslant\left(1-\alpha_{n}\right)^{2}\left\|y_{n}-p\right\|^{2}+2 \alpha_{n}\left\langle f\left(x_{n}\right)-p, x_{n+1}-p\right\rangle \\
& \leqslant\left(1-\alpha_{n}\right)^{2}\left\|z_{n}-p\right\|^{2}+2 \alpha_{n}\left\langle f\left(x_{n}\right)-p, x_{n+1}-p\right\rangle \\
& \leqslant\left(1-\alpha_{n}\right)^{2}\left(\left\|x_{n}-p\right\|^{2}-\left\|x_{n}-u_{n}\right\|^{2}\right)+2 \alpha_{n}\left\|f\left(x_{n}\right)-p\right\|\left\|x_{n+1}-p\right\| \\
& \leqslant\left(1-2 \alpha_{n}+\alpha_{n}^{2}\right)\left\|x_{n}-p\right\|^{2}-\left(1-\alpha_{n}\right)^{2}\left\|x_{n}-u_{n}\right\|^{2}+2 \alpha_{n}\left\|f\left(x_{n}\right)-p\right\|\left\|x_{n+1}-p\right\| \\
& \leqslant\left\|x_{n}-p\right\|^{2}+\alpha_{n}\left\|x_{n}-p\right\|^{2}-\left(1-\alpha_{n}\right)^{2}\left\|x_{n}-u_{n}\right\|^{2}+2 \alpha_{n}\left\|f\left(x_{n}\right)-p\right\|\left\|x_{n+1}-p\right\|,
\end{aligned}
$$

and hence

$$
\left(1-\alpha_{n}\right)^{2}\left\|x_{n}-u_{n}\right\|^{2} \leqslant\left\|x_{n}-p\right\|^{2}-\left\|x_{n+1}-p\right\|^{2}+\alpha_{n}\left\|x_{n}-p\right\|^{2}+2 \alpha_{n}\left\|f\left(x_{n}\right)-p\right\|\left\|x_{n+1}-p\right\| .
$$

Thanks to inequality (3.5), $\left\{x_{n}\right\}$ being bounded, and $\alpha_{n} \rightarrow 0$ as $n \rightarrow \infty$, we have

$$
\lim _{n \rightarrow \infty}\left\|x_{n}-u_{n}\right\|=0 .
$$


Also, from (3.8) and (3.10), we have

$$
\lim _{n \rightarrow \infty}\left\|x_{n}-z_{n}\right\|=0
$$

Letting $p \in \Gamma$, we have

$$
\begin{aligned}
\left\|v_{n}-p\right\|^{2}=\left\|T_{r_{n}} z_{n}-T_{r_{n}} p\right\|^{2} & \leqslant\left\langle T_{r_{n}} z_{n}-T_{r_{n}} p, z_{n}-p\right\rangle \\
& \leqslant\left\langle v_{n}-p, z_{n}-p\right\rangle=\frac{1}{2}\left(\left\|v_{n}-p\right\|^{2}+\left\|z_{n}-p\right\|^{2}-\left\|z_{n}-v_{n}\right\|^{2}\right)
\end{aligned}
$$

and hence

$$
\left\|v_{n}-p\right\|^{2} \leqslant\left\|z_{n}-p\right\|^{2}-\left\|z_{n}-v_{n}\right\|^{2}
$$

Therefore, from (3.1) and (3.11), we obtain

$$
\begin{aligned}
\left\|x_{n+1}-p\right\|^{2}= & \left\|\alpha_{n} f\left(x_{n}\right)+\left(1-\alpha_{n}\right) y_{n}-p\right\|^{2} \\
\leqslant & \left(1-\alpha_{n}\right)^{2}\left\|y_{n}-p\right\|^{2}+2 \alpha_{n}\left\langle f\left(x_{n}\right)-p, x_{n+1}-p\right\rangle \\
\leqslant & \left(1-\alpha_{n}\right)^{2}\left\|v_{n}-p\right\|^{2}+2 \alpha_{n}\left\langle f\left(x_{n}\right)-p, x_{n+1}-p\right\rangle \\
\leqslant & \left(1-\alpha_{n}\right)^{2}\left\|v_{n}-p\right\|^{2}+2 \alpha_{n}\left\langle f\left(x_{n}\right)-f(p), x_{n+1}-p\right\rangle+2 \alpha_{n}\left\langle f(p)-p, x_{n+1}-p\right\rangle \\
\leqslant & \left(1-\alpha_{n}\right)^{2}\left(\left\|z_{n}-p\right\|^{2}-\left\|z_{n}-v_{n}\right\|^{2}\right)+2 \alpha_{n} b\left\|x_{n}-p\right\|\left\|x_{n+1}-p\right\|+2 \alpha_{n}\|f(p)-p\|\left\|x_{n+1}-p\right\| \\
\leqslant & \left(1-2 \alpha_{n}+\alpha_{n}^{2}\right)\left\|x_{n}-p\right\|^{2}-\left(1-\alpha_{n}\right)^{2}\left\|z_{n}-v_{n}\right\|^{2} \\
& +2 \alpha_{n} b\left\|x_{n}-p\right\|\left\|x_{n+1}-p\right\|+2 \alpha_{n}\|f(p)-p\|\left\|x_{n+1}-p\right\| \\
\leqslant & \left\|x_{n}-p\right\|^{2}+\alpha_{n}\left\|x_{n}-p\right\|^{2}-\left(1-\alpha_{n}\right)^{2}\left\|z_{n}-v_{n}\right\|^{2}+2 \alpha_{n} b\left\|x_{n}-p\right\|\left\|x_{n+1}-p\right\| \\
& +2 \alpha_{n}\|f(p)-p\|\left\|x_{n+1}-p\right\|
\end{aligned}
$$

and hence

$$
\begin{aligned}
\left(1-\alpha_{n}\right)^{2}\left\|z_{n}-v_{n}\right\|^{2} \leqslant & \left\|x_{n}-p\right\|^{2}-\left\|x_{n+1}-p\right\|^{2}+\alpha_{n}\left\|x_{n}-p\right\|^{2}+2 \alpha_{n} b\left\|x_{n}-p\right\|\left\|x_{n+1}-p\right\| \\
& +2 \alpha_{n}\|f(p)-p\|\left\|x_{n+1}-p\right\| .
\end{aligned}
$$

So, we have

$$
\lim _{n \rightarrow \infty}\left\|z_{n}-v_{n}\right\|=0 \text {. }
$$

Now, we prove that $\limsup _{n \rightarrow+\infty}\left\langle x^{*}-f\left(x^{*}\right), x^{*}-x_{n}\right\rangle \leqslant 0$. Since $H$ is reflexive and $\left\{x_{n}\right\}$ is bounded, there exists a sub-sequence $\left\{x_{n_{k}}\right\}$ of $\left\{x_{n}\right\}$ which converges weakly to $\omega$ in $K$ and

$$
\limsup _{n \rightarrow+\infty}\left\langle x^{*}-f\left(x^{*}\right), x^{*}-x_{n}\right\rangle=\lim _{k \rightarrow+\infty}\left\langle x^{*}-f\left(x^{*}\right), x^{*}-x_{n_{k}}\right\rangle .
$$

From (3.6), (3.12), and I - T being demiclosed, we obtain $\omega \in \operatorname{Fix}(T)$. Moreover, note that by (A2) and Lemma 2.13, we have

$$
\frac{1}{r_{n}}\left\langle y-v_{n}, v_{n}-z_{n}\right\rangle \geqslant g\left(y, v_{n}\right)
$$

and hence

$$
\left\langle y-v_{n_{k}}, \frac{v_{n_{k}}-z_{n_{k}}}{r_{n_{k}}}\right\rangle \geqslant g\left(y, v_{n_{k}}\right) .
$$

Since $\frac{v_{n_{k}}-z_{n_{k}}}{r_{n_{k}}} \rightarrow 0$ and $v_{n_{k}} \rightarrow \omega$, it follows from (A4) that $g(y, \omega) \leqslant 0$ for all $y \in K$. For $t$ with $0<t<1$ and $y \in K$, let $y_{t}=t y+(1-t) \omega$. Since $y \in \omega$ and $\omega \in K$, we have $y_{t} \in K$ and hence $g\left(y_{t}, \omega\right) \leqslant 0$. So, from (A1) and (A4) we have

$$
0=g\left(y_{t}, y_{t}\right) \leqslant \operatorname{tg}\left(y_{t}, y\right)+(1-t) g\left(y_{t}, w\right) \leqslant \operatorname{tg}\left(y_{t}, y\right)
$$


and hence $0 \leqslant g\left(y_{t}, y\right)$. From $(A 3)$, we have $g(\omega, y) \geqslant 0$ for all $y \in K$ and hence $\omega \in E P(g)$. Using (3.1) and Lemma 2.10, we arrive at

$$
\begin{aligned}
\left\|x_{n}-J_{\beta}^{F} x_{n}\right\| & \leqslant\left\|u_{n}-J_{\beta}^{F} x_{n}\right\|+\left\|u_{n}-x_{n}\right\| \\
& \leqslant\left\|J_{\lambda_{n}}^{F} x_{n}-J_{\beta}^{F} x_{n}\right\|+\left\|u_{n}-x_{n}\right\| \\
& \leqslant\left\|u_{n}-x_{n}\right\|+\left\|J_{\beta}^{F}\left(\frac{\lambda_{n}-\beta}{\lambda_{n}} J_{\lambda_{n}}^{F} x_{n}+\frac{\beta}{\lambda_{n}} x_{n}\right)-J_{\beta}^{F} x_{n}\right\| \\
& \leqslant\left\|u_{n}-x_{n}\right\|+\left\|\frac{\lambda_{n}-\beta}{\lambda_{n}} J_{\lambda_{n}}^{F} x_{n}+\frac{\beta}{\lambda_{n}} x_{n}-x_{n}\right\| \\
& \leqslant\left\|u_{n}-x_{n}\right\|+\left(1-\frac{\beta}{\lambda_{n}}\right)\left\|u_{n}-x_{n}\right\| \\
& \leqslant\left(2-\frac{\beta}{\lambda_{n}}\right)\left\|u_{n}-x_{n}\right\| .
\end{aligned}
$$

Hence,

$$
\lim _{n \rightarrow \infty}\left\|x_{n}-J_{\beta}^{F} x_{n}\right\|=0
$$

Since $\mathrm{J}_{\beta}^{\mathrm{F}}$ is single valued and non-expansive, using (3.13) and Lemma 2.2, then

$$
\omega \in \operatorname{Fix}\left(J_{\beta}^{F}\right)=\operatorname{argmin}_{u \in K} F(u) .
$$

Let us show $\omega \in \operatorname{VI}(A, K)$. Now, let us introduce the multi-valued map $B: H \rightarrow 2^{H}$ defined by:

$$
\mathrm{B} z= \begin{cases}\mathrm{A} z+\mathrm{N}_{\mathrm{K}}(z), & \text { if } z \in \mathrm{K}, \\ \emptyset, & \text { if } z \notin \mathrm{K}\end{cases}
$$

where $\mathrm{N}_{\mathrm{K}}(z)$ is the normal $\mathrm{K}$ at $z$ and is defined as follows:

$$
\mathrm{N}_{\mathrm{K}}(z)=\{w \in \mathrm{H}:\langle w, z-v\rangle \geqslant 0, \forall v \in \mathrm{K}\} .
$$

From Lemma 2.7, we have that $B$ is maximal monotone and $B^{-1}(0)=\operatorname{VI}(A, K)$. Let $(u, v) \in G(A)$. Since $v-A u \in N_{K}(u)$ and $z_{\mathfrak{n}} \in K$, we have

$$
\left\langle u-z_{n}, v-A u\right\rangle \geqslant 0 .
$$

On other hand, from $z_{n}=P_{K}\left(I-\theta_{n} A\right) u_{n}$, we have, $\left\langle u-z_{n}, z_{n}-\left(I-\theta_{n} A\right) u_{n}\right\rangle \geqslant 0$ and hence

$$
\left\langle u-z_{n}, \frac{z_{n}-u_{n}}{\theta_{n}}+A u_{n}\right\rangle \geqslant 0 .
$$

Therefore, we have

$$
\begin{aligned}
\left\langle u-z_{n_{k}}, v\right\rangle & \geqslant\left\langle u-z_{n_{k}}, A u\right\rangle \\
& \geqslant\left\langle u-z_{n_{k}}, A u\right\rangle-\left\langle u-z_{n_{k}}, \frac{z_{n_{k}}-u_{n_{k}}}{\theta_{n_{k}}}+A u_{n_{k}}\right\rangle \\
& \geqslant\left\langle u-z_{n_{k}}, A u-A z_{n_{k}}\right\rangle+\left\langle u-z_{n_{k}}, A z_{n_{k}}-A u_{n_{k}}\right\rangle-\left\langle u-z_{n_{k}}, \frac{z_{n_{k}}-u_{n_{k}}}{\theta_{n_{k}}}\right\rangle \\
& \geqslant\left\langle u-z_{n_{k}}, A z_{n_{k}}-A u_{n_{k}}\right\rangle-\left\langle u-z_{n_{k}}, \frac{z_{n_{k}}-u_{n_{k}}}{\theta_{n_{k}}}\right\rangle .
\end{aligned}
$$

By using A being $\frac{1}{\alpha}$ Lipschitz, we have

$$
\left\langle u-z_{n_{k}}, v\right\rangle \geqslant-M\left(\frac{\left\|z_{n_{k}}-u_{n_{k}}\right\|}{\alpha}+\frac{\left\|z_{n_{k}}-u_{n_{k}}\right\|}{a}\right),
$$


where $M$ is a positive constant such that $\sup _{k \geqslant 1}\left\{\left\|u-z_{n_{k}}\right\|\right\} \leqslant M$. Since $z_{n_{k}} \rightarrow \omega$, it follows from (3.8) that $\langle u-\omega, v\rangle \geqslant 0$ as $k \rightarrow \infty$. Since $B$ is maximal monotone, we have $\omega \in B^{-1}(0)$ and we obtain that $\omega \in \operatorname{VI}(A, K)$. Therefore, $\omega \in \Gamma$. Hence

$$
\begin{aligned}
\limsup _{n \rightarrow+\infty}\left\langle x^{*}-f\left(x^{*}\right), x^{*}-x_{n}\right\rangle & =\lim _{k \rightarrow+\infty}\left\langle x^{*}-f\left(x^{*}\right), x^{*}-x_{n_{k}}\right\rangle \\
& =\left\langle x^{*}-f\left(x^{*}\right), x^{*}-\omega\right\rangle \leqslant 0 .
\end{aligned}
$$

Finally, we show that $x_{n} \rightarrow x^{*}$. From (3.1), we have

$$
\begin{aligned}
\left\|x_{n+1}-x^{*}\right\|^{2} & =\left\|\alpha_{n} f\left(x_{n}\right)+\left(1-\alpha_{n}\right) y_{n}-x^{*}\right\|^{2} \\
& \leqslant\left\|\alpha_{n}\left(f\left(x_{n}\right)-f\left(x^{*}\right)\right)+\left(1-\alpha_{n}\right)\left(y_{n}-x^{*}\right)\right\|^{2}+2 \alpha_{n}\left\langle x^{*}-f\left(x^{*}\right), x^{*}-x_{n+1}\right\rangle \\
& \leqslant\left(\alpha_{n}\left\|f\left(x_{n}\right)-f\left(x^{*}\right)\right\|+\left\|\left(1-\alpha_{n}\right)\left(y_{n}-x^{*}\right)\right\|\right)^{2}+2 \alpha_{n}\left\langle x^{*}-f\left(x^{*}\right), x^{*}-x_{n+1}\right\rangle \\
& \leqslant\left(\alpha_{n} b\left\|x_{n}-x^{*}\right\|+\left(1-\alpha_{n}\right)\left\|y_{n}-x^{*}\right\|\right)^{2}+2 \alpha_{n}\left\langle x^{*}-f\left(x^{*}\right), x^{*}-x_{n+1}\right\rangle \\
& \leqslant\left(\left(1-\alpha_{n}(1-b)\right)\left\|x_{n}-x^{*}\right\|\right)^{2}+2 \alpha_{n}\left\langle x^{*}-f\left(x^{*}\right), x^{*}-x_{n+1}\right\rangle \\
& \leqslant\left(1-\alpha_{n}(1-b)\right)\left\|x_{n}-x^{*}\right\|^{2}+2 \alpha_{n}\left\langle x^{*}-f\left(x^{*}\right), x^{*}-x_{n+1}\right\rangle .
\end{aligned}
$$

From Lemma 2.4, its follows that $x_{n} \rightarrow x^{*}$.

Case 2. Assume that the sequence $\left\{\left\|x_{n}-x^{*}\right\|\right\}$ is not monotonically decreasing. Set $B_{n}=\left\|x_{n}-x^{*}\right\|$ and $\tau: \mathbb{N} \rightarrow \mathbb{N}$ be a mapping for all $n \geqslant n_{0}$ (for some $n_{0}$ large enough) by $\tau(n)=\max \left\{k \in \mathbb{N}: k \leqslant n, B_{k} \leqslant\right.$ $\left.B_{k+1}\right\}$. We have $\tau$ is a non-decreasing sequence such that $\tau(n) \rightarrow \infty$ as $n \rightarrow \infty$ and $B_{\tau(n)} \leqslant B_{\tau(n)+1}$ for $n \geqslant n_{0}$. From (3.4), we have

$$
\left(1-\alpha_{\tau(n)}\right)\left(1-\beta_{\tau(n)}\right)\left(\beta_{\tau(n)}-\lambda\right)\left\|\nu_{\tau(n)}-T \nu_{\tau(n)}\right\|^{2} \leqslant 2 \alpha_{\tau(n)} C \rightarrow 0 \text { as } n \rightarrow \infty .
$$

Since $\left.\beta_{n} \in\right] \lambda, 1\left[\right.$ and $\lim _{n \rightarrow \infty} \inf \left(1-\beta_{\tau(n)}\right)\left(\beta_{\tau(n)}-\lambda\right)>0$, we can deduce

$$
\lim _{n \rightarrow \infty}\left\|v_{\tau(n)}-T v_{\tau(n)}\right\|=0
$$

By a similar argument as in case 1 , we can show that $x_{\tau(n)}$ and $\nu_{\tau(n)}$ are bounded in $K$ and $\limsup _{\tau(n) \rightarrow+\infty}\left\langle x^{*}-\right.$ $\left.f\left(x^{*}\right), x^{*}-x_{\tau(n)}\right\rangle \leqslant 0$. We have for all $n \geqslant n_{0}$,

$$
0 \leqslant\left\|x_{\tau(n)+1}-x^{*}\right\|^{2}-\left\|x_{\tau(n)}-x^{*}\right\|^{2} \leqslant \alpha_{\tau(n)}\left[-(1-b)\left\|x_{\tau(n)}-x^{*}\right\|^{2}+2\left\langle x^{*}-f\left(x^{*}\right), x^{*}-x_{\tau(n)+1}\right\rangle,\right.
$$

which implies that

$$
\left\|x_{\tau(n)}-x^{*}\right\|^{2} \leqslant \frac{2}{1-b}\left\langle x^{*}-f\left(x^{*}\right), x^{*}-x_{\tau(n)+1}\right\rangle .
$$

Then, we have

$$
\lim _{n \rightarrow \infty}\left\|x_{\tau(n)}-x^{*}\right\|^{2}=0
$$

Therefore,

$$
\lim _{n \rightarrow \infty} B_{\tau(n)}=\lim _{n \rightarrow \infty} B_{\tau(n)+1}=0 .
$$

Thus, by Lemma 2.9, we conclude that

$$
0 \leqslant B_{n} \leqslant \max \left\{B_{\tau(n)}, B_{\tau(n)+1}\right\}=B_{\tau(n)+1} .
$$

Hence, $\lim _{n \rightarrow \infty} B_{n}=0$, that is $\left\{x_{n}\right\}$ converges strongly to $x^{*}$. This completes the proof. 
We apply Theorem 3.1 when we consider fixed point problems involving strictly pseudo-contractive mappings. In this case demiclosedness assumption is not necessary.

Theorem 3.2. Let $\mathrm{K}$ be a nonempty, closed convex subset of a real Hilbert space $\mathrm{H}$. Let $\mathrm{A}: \mathrm{K} \rightarrow \mathrm{H}$ be an $\alpha$-inverse strongly monotone operator and let $\mathrm{g}$ be a bifunction from $\mathrm{K} \times \mathrm{K} \rightarrow \mathbb{R}$ satisfying (A1)-(A4). Let $\mathrm{f}: \mathrm{K} \rightarrow \mathrm{K}$ be a contraction with coefficient $\mathrm{b}$ and $\mathrm{F}: \mathrm{K} \rightarrow(-\infty,+\infty]$ be a proper convex and lower semi-continuous function. Let $\mathrm{T}: \mathrm{K} \rightarrow \mathrm{K}$ be a $\lambda$-strictly pseudo-contractive mapping such that $\Gamma:=\operatorname{Fix}(\mathrm{T}) \cap \mathrm{VI}(\mathrm{A}, \mathrm{K}) \cap \operatorname{argmin}_{\mathrm{u} \in \mathrm{K}} \mathrm{F}(\mathrm{u}) \cap$ $\mathrm{EP}(\mathrm{g}) \neq \emptyset$. Let $\left\{x_{\mathrm{n}}\right\}$ be a sequence defined as follows:

$$
\left\{\begin{array}{l}
x_{0} \in K, \\
u_{n}=\operatorname{argmin}_{u \in K}\left[F(u)+\frac{1}{2 \lambda_{n}}\left\|u-x_{n}\right\|^{2}\right], \\
z_{n}=P_{K}\left(I-\theta_{n} A\right) u_{n}, \\
g\left(v_{n}, y\right)+\frac{1}{r_{n}}\left\langle y-v_{n}, v_{n}-z_{n}\right\rangle \geqslant 0, \forall y \in K, \\
y_{n}=\beta_{n} v_{n}+\left(1-\beta_{n}\right) T v_{n}, \\
x_{n+1}=\alpha_{n} f\left(x_{n}\right)+\left(1-\alpha_{n}\right) y_{n},
\end{array}\right.
$$

where $\left.\beta_{n} \in\right] \lambda, 1\left[,\left\{\alpha_{n}\right\} \subset(0,1),\left\{\theta_{n}\right\} \subset(0,1)\right.$ and $\left.\left\{r_{n}\right\} \subset\right] 0, \infty$ [ satisfy the following conditions; $\lim _{n \rightarrow \infty} \alpha_{n}=0$, $\sum_{n=0}^{\infty} \alpha_{n}=\infty, \lim _{n \rightarrow \infty} \inf \left(1-\beta_{n}\right)\left(\beta_{n}-\lambda\right)>0, \theta_{n} \in[a, b] \subset(0, \min \{1,2 \alpha\}), \lim _{n \rightarrow \infty} \inf _{n}>0$ and $\left\{\lambda_{n}\right\}$ is a sequence such that $\lambda_{n} \geqslant \beta>0$ for all $n \geqslant 1$ and some $\beta$. Then, the sequence $\left\{x_{n}\right\}$ generated by (3.14) converges strongly to $x^{*} \in \Gamma$, which is the unique solution of variational inequality (3.2).

Proof. Since every strictly pseudo-contractive mapping is demicontractive, then, the proof follows from Lemma 2.6 and Theorem 3.1.

\section{Numerical example}

In this section, we present a numerical example to illustrate the convergence behavior of our iteration scheme (3.1). Let $H=\mathbb{R}$ and $K=[0,1]$. We set $F(x):=\frac{1}{2}\|x\|^{2}, g(x, y):=y^{2}+y x-2 x^{2}, \quad T x=\frac{1}{2} x$, $f(x)=\frac{1}{3} x$ and $A \equiv 0$. It can easily be seen that $F, g, A$, and $T$ are satisfied the conditions in Theorem 3.1. We can observe that $T_{r}(x)=\frac{1}{1+3 r} x$ and $\operatorname{Fix}(T) \cap V I(A, K) \cap \operatorname{argmin}_{u \in K} F(u) \cap E P(g)=\{0\}$. Using the proximity operator [6], we know that

$$
\operatorname{argmin}_{\mathfrak{u} \in \mathrm{K}}\left[\mathrm{F}(\mathrm{u})+\frac{1}{2}\|\mathrm{u}-x\|^{2}\right]=\operatorname{prox}_{\mathrm{F}} x=\frac{x}{2} .
$$

We take $r=1, \alpha_{n}=\frac{1}{n}$ and $\beta_{n}=\frac{1}{2 n}+\frac{1}{2}$. Then, the scheme (3.1) can be simplified as

$$
\left\{\begin{array}{l}
u_{n}=\frac{x_{n}}{2} \\
v_{n}=\frac{x_{n}}{8} \\
y_{n}=\frac{n+1}{16 n} x_{n}+\frac{n+1}{16 n} \\
x_{n+1}=\frac{1}{3 n} x_{n}+\frac{n-1}{n} y_{n}, n \geqslant 1
\end{array}\right.
$$

Take the initial point $x_{1}=1$, the numerical experiment result using SciLab is given by Figure 1, which shows the iteration process of the sequence $x_{n}$ converges strongly to 0 . 


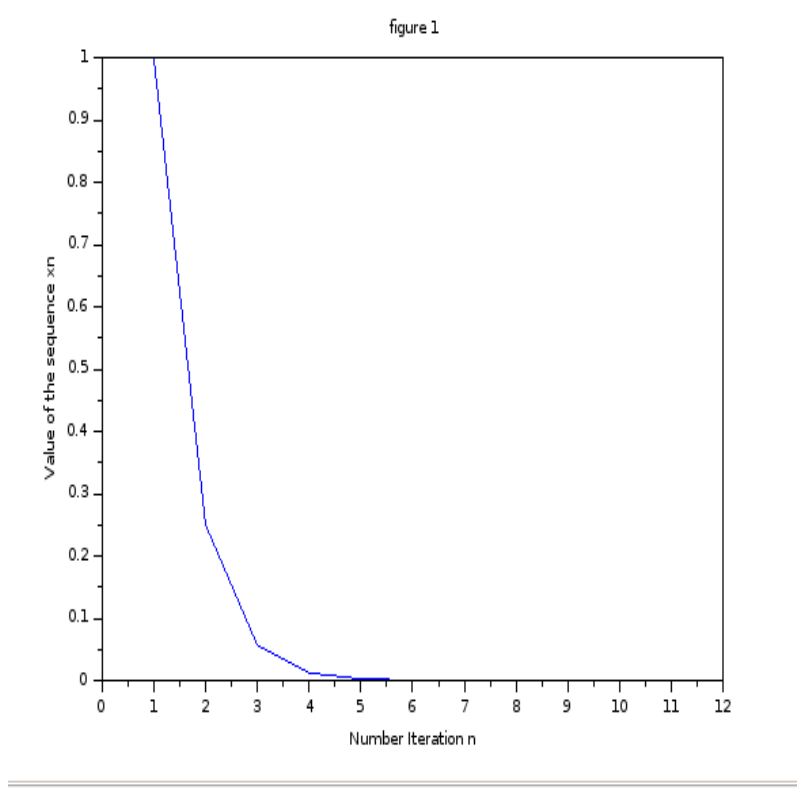

Figure 1

\section{Conclusion}

In this work, we proposed a new iteration scheme for the approximation of a common element of the set of minimizers of a convex function, the set of solutions of variational inequality problem, the set of solutions of equilibrium problems and the set of fixed points of demicontractive mappings. The results obtained in this paper are important improvements of recent important results in nonlinear analysis. It is of worth interest to establish such results in general Banach spaces.

\section{References}

[1] L. Ambrosio, N. Gigli, G. Savaré, Gradient flows in metric spaces and in the space of probability measures, Birkhäuser Verlag, Basel, (2008).

[2] P. N. Anh, L. T. H. An, The subgradient extragradient method extended to equilibrium problems, Optimization, 64 (2015), 225-248. 1

[3] E. Blum, W. Oettli, From optimization and variational inequalities to equilibrium problems, Math. Student, 63 (1994), 123-145. 1, 2, 2.12

[4] C. Chidume, Geometric Properties of Banach spaces and Nonlinear Iterations, Springer-Verlag London, London, (2009). $1,2.3$

[5] C. E. Chidume, N. Djitté, Strong convergence theorems for zeros of bounded maximal monotone nonlinear operators, Abstr. Appl. Anal., 2012 (2012), 19 pages. 1

[6] P. L. Combettes, J. C. Pesquet, Proximal splitting methods in signal processing, in: Fixed-Point Algorithms for Inverse Problems in Science and Engineering, 2011 (2011), 185-212. 4

[7] K. Fan, A minimax inequality and applications, in: Inequalities III, 1972 (1972), 103-113.

[8] O. Güler, On the convergence of the proximal point algorithm for convex minimization, SIAM J. Control Optim., 29 (1991), 403-419. 1, 2

[9] H. Iiduka, W. Takahashi, M. Toyoda, Approximation of solutions of variational inequalities for monotone mappings, PanAmer. Math. J., 14 (2004), 49-61. 1

[10] S. Kamimura, W. Takahashi, Strong convergence of a proximal-type algorithm in a Banach space, SIAM J. Optim., 13 (2002), 938-945. 1

[11] J. L. Lions, G. Stampacchia, Variational inequalities, Comm. Pure Appl. Math., 20 (1967), 493-519. 1

[12] P.-E. Maingé, A hybrid extragradient-viscosity method for monotone operators and fixed point problems, SIAM J. Control Optim., 47 (2008), 1499-1515. 1

[13] P.-E. Maingé, Strong convergence of projected subgradient methods for nonsmooth and nonstrictly convex minimization, Set-Valued Anal., 16 (2008), 899-912. 2.9, 2.11

[14] W. A. Mann, Mean value methods in iteration, Proc. Amer. Math. Soc., 4 (1953), 506-510. 
[15] G. Marino, B. Scardamaglia, R. Zaccone, A general viscosity explicit midpoint rule for quasi-nonexpansive mappings, J. Nonlinear Convex Anal., 18 (2017), 137-148.

[16] G. Marino, H.-K. Xu, A general iterative method for nonexpansive mappings in Hibert spaces, J. Math. Anal. Appl., 318 (2006), 43-52. 1

[17] G. Marino, H.-K. Xu, Weak and strong convergence theorems for strict pseudo-contractions in Hilbert spaces, J. Math. Math. Appl., 329 (2007), 336-346. 2.5, 2.6

[18] B. Martinet, Regularisation, d'inéquations variationnelles par approximations successives, Rev. Franaise Informat. Recherche Oprationnelle, 4 (1970), 154-158. 1

[19] I. Miyadera, Nonlinear semigroups, American Mathematical Society, Providence, (1992). 2.10

[20] A. Moudafi, Viscosity approximation methods for fixed point problems, J. Math. Anal. Appl., 241 (2000), 46-55. 1, 1

[21] N. Petrot, K. Wattanawitoon, P. Kumam, A hybrid projection method for generalized mixed equilibrium problems and fixed point problems in Banach spaces, Nonlinear Anal. Hybrid Syst., 4 (2010), 631-643. 1

[22] X. L. Qin, Y. J. Cho, S. M. Kang, H. Zhou, Convergence of a modified Halpern-type iteration algorithm for quasi- $\phi-$ nonexpansive mappings, Appl. Math. Lett., 22 (2009), 1051-1055. 1

[23] R. T. Rockafellar, On the maximality of sums of nonlinear monotone operators, Trans. Amer. Math. Soc., 149 (1970), 75-88. 1, 1, 2.2, 2.7

[24] T. M. M. Sow, An iterative algorithm for solving equilibrium problems, variational inequality problems and fixed point problems with multivalued quasi-nonexpansive mappings, Appl. Set-Valued Anal. Optim., 1 (2019), 171-185. 1, 2.8

[25] S. Takahashi, W. Takahashi, Viscosity approximation methods for equilibrium problems and fixed point problems in Hilbert spaces, J. Math. Anal. Appl., 331 (2007), 506-515. 1

[26] W. Takahashi, M. Toyoda, Weak convergence theorems for nonexpansive mappings and monotone mappings, J. Optim. Theory Appl., 118 (2003), 417-428.

[27] M. Tian, B.-N. Jiang, Weak convergence theorem for zero points of inverse strongly monotone mapping and fixed points of nonexpansive mapping in Hilbert space, Optimization, 66 (2017), 1689-1698. 1

[28] M. Tian, M. Tong, A self-adaptive Armijo-like step size method for solving monotone variational inequality problems in Hilbert spaces, J. Nonlinear Funct. Anal., 2019 (2019), 15 pages. 1

[29] S. Wang, A general iterative method for an infinite family of strictly pseudo-contractive mappings in Hilbert spaces, Appl. Math. Lett., 24 (2011), 901-907.

[30] H.-K. Xu, Inequalities in Banach spaces with applications, Nonlinear Anal., 16 (1991), 1127-1138. 1

[31] H.-K. Xu, Iterative algorithms for nonlinear operators, J. London Math. Soc. (2), 66 (2002), 240-256. 1, 2.4

[32] H.-K. Xu, A variable Krasnoselskii-Mann algorithm and the multiple set split feasiblity problem, Inverse Problem, 26 (2006), 2021-2034. 2, 2.13

[33] H.-K. Xu, Iterative methods for the split feasiblity problem in infinite-dimensional Hilbert spaces, Inverse Problem, 26 (2010), 1-17. 1

[34] H.-K. Xu, M. A. Alghamdi, N. Shahzad, The viscosity technique for the implicit midpoint rule of nonexpansive mappings in Hilbert spaces, Fixed Point Theory Appl., 2015 (2015), 12 pages.

[35] Y. Yao, H. Zhou, Y. C. Liou, Strong convergence of modified Krasnoselskii-Mann iterative algorithm for nonexpansive mappings, J. Math. Anal. Appl. Comput., 29 (2009), 383-389. 1 